\title{
ON THE DIRICHLET PROBLEM OF ELLIPTIC TYPE
}

\author{
MAREK GALEWSKI
}

We investigate the existence of solutions and their stability for elliptic Dirichlet problems with nonlinearity of a convex-concave type. By relating the primal action and the dual action functionals on certain subsets of their domains we get the existence of solutions which are further stable with respect to a numerical parameter. We allow also for the differential operator to depend on a numerical parameter.

\section{INTRODUCTION}

In the paper we consider the existence and stability of solutions the following family of boundary value problems for $k=0,1,2, \ldots$

$$
\begin{gathered}
-\operatorname{div}\left(\varphi_{k}\left(y,|\nabla x|^{n-1}\right)|\nabla x|^{n-2} \nabla x\right)+H_{x}^{k}(y, x)=G_{x}^{k}(y, x) \\
\left.x(y)\right|_{\partial \Omega}=0, x \in W_{0}^{1, n}(\Omega)
\end{gathered}
$$

$G_{x}^{k}(y, x) \rightarrow G_{x}^{0}(y, x), H_{x}^{k}(y, x) \rightarrow H_{x}^{0}(y, x), \varphi_{k}(y, x) \rightarrow \varphi_{0}(y, x)$ almost everywhere on $\Omega$, for all $x$ in a certain interval that will be specified later, $1 / n+1 / m=1, \varphi_{k}: \Omega \times R \rightarrow R$ is a Caratheodory function, that is, it is continuous with respect to $x$ for almost everywhere $y \in \Omega$ and measurable in $y$ for every $x \in R$; there exist constants $M_{1}^{k}, M_{2}^{k}>0$ such that for almost everywhere $y \in \Omega$ and for all $t \in R_{+}$

$$
M_{1}^{k} \leqslant \varphi_{k}(y, t) \leqslant M_{2}^{k}
$$

and such that the sequences $\left\{M_{1}^{k}\right\}_{k=0}^{\infty},\left\{M_{2}^{k}\right\}_{k=0}^{\infty}$ are bounded by $M_{1}$ and $M_{2}$ say; $\Omega \subset R^{r}$ is a region with a regular boundary, $n>r$; there exist constants $m_{k} \geqslant m_{0}>0$ such that for all $a \geqslant b, a, b \in R$ and almost everywhere $y \in \Omega$

$$
\varphi_{k}(y, a) a-\varphi_{k}(y, b) b \geqslant m_{k}(a-b)
$$

Let $\left\{d_{k}\right\}_{k=1}^{\infty}$ be a sequence of decreasing positive numbers bounded away from 0 . Let $c_{S}, C$ denote the best Sobolev constants from the inequalities

$$
\max _{y \in \Omega}|x(y)| \leqslant c_{s}\|\nabla x\|_{L^{n}(\Omega)},\|x\|_{L^{n}(\Omega)} \leqslant C\|\nabla x\|_{L^{n}(\Omega)}
$$

Received 12th June, 2006

Copyright Clearance Centre, Inc. Serial-fee code: 0004-9727/07 \$A2.00+0.00. 
By $\left(H^{k}\right)^{*},\left(G^{k}\right)^{*}: \Omega \times R \rightarrow R$ we understand the Fenchel-Young duals of functions $H^{k}$, $G^{k}: \Omega \times R \rightarrow R$ with respect to the second variable defined by, see [1]

$$
\left(H^{k}\right)^{*}(y, u)=\sup _{x \in R}\left\{x u-H^{k}(y, x)\right\},\left(G^{k}\right)^{*}(y, u)=\left\{\sup _{x \in R} x u-G^{k}(y, x)\right\} .
$$

We assume for all $k=0,1,2, \ldots$ that

G1. $G^{k}, G_{x}^{k}: \Omega \times\left[-d_{0}, d_{0}\right] \rightarrow R$ are Caratheodory functions, $G^{k}$ is convex with respect to $x$ for almost everywhere $y, G^{k}(y, x)=+\infty$ for $(y, x) \in \Omega \times\left(R \backslash\left[-d_{0}, d_{0}\right]\right)$ and

$$
\frac{(\operatorname{vol}(\Omega))^{1 / m} c_{S}^{n-1}}{M_{1}^{k}} C \max _{x \in\left[-d_{0}, d_{0}\right]}\left|G_{x}^{k}(y, x)\right| \leqslant d_{k}^{n-1} .
$$

G2. $G_{x}^{k}(y, 0) \neq 0$, for almost everywhere $y \in \Omega$, function $y \rightarrow\left(G^{k}\right)^{*}(y, 0)$ is integrable. G3. $G_{x}^{k}$ is differentiable in $x$ on $\left[-d_{0}, d_{0}\right]$ for almost everywhere $y \in \Omega$ and there exists a constant $a>0$ (independent of $k$ ) such that

$$
\max _{x \in\left[-d_{0}, d_{0}\right]}\left|G_{x x}^{k}(y, x)\right| \leqslant a
$$

H1. $H^{k}, H_{x}^{k}: \Omega \times R \rightarrow R$ are Caratheodory function, $H^{k}$ is convex with respect to the second variable,

$$
H_{x}^{k}(y, x) x \geqslant 0 \text { for } x \in R, H_{x}^{k}(y, 0)=0, \text { for almost everywhere } y \in \Omega,
$$

function $y \rightarrow\left(H^{k}\right)^{*}(y, 0)$ is integrable, there exists a constant $\beta$ (independent of $k$ )

$$
\max _{x \in\left[-d_{0}, d_{0}\right]}\left|H_{x}^{k}(y, x)\right| \leqslant \beta
$$

H2. $H_{x}^{k}$ is differentiable in $x$ on $\left[-d_{k}, d_{k}\right]$ for almost everywhere $y \in \Omega$ and there exists a constant $b>0$ (independent of $k$ ) such that

$$
\max _{x \in\left[-d_{0}, d_{0}\right]}\left|H_{x x}^{k}(y, x)\right| \leqslant b .
$$

Let us define for all $k=0,1,2, \ldots$ the following number

$$
g_{k}=\operatorname{essesup} \max _{y \in \Omega}\left|G_{x \in\left[-d_{0}, d_{0}\right]}^{k}(y, x)\right| .
$$

We prove that for each $k=1,2, \ldots$ there exists a solution $x_{k}$ to problem (1.1) satisfying: $x_{k}(y) \in\left[-d_{k}, d_{k}\right]$ on $\Omega$ and $\left\|\nabla x_{k}\right\|_{L^{n}(\Omega)}^{n-1} \leqslant C(\operatorname{vol}(\Omega))^{1 / m} /\left(M_{1}^{k}\right) g_{k}$. Moreover from a sequence $\left\{x_{k}\right\}_{k=1}^{\infty}$ we may choose a subsequence $\left\{x_{k_{j}}\right\}_{j=1}^{\infty}$ such that such that $\lim _{j \rightarrow \infty} x_{k_{j}}=\bar{x}$ weakly in $W_{0}^{1, n}(\Omega)$ and

$$
\begin{gathered}
-\operatorname{div}\left(\varphi_{0}\left(y,|\nabla \bar{x}|^{n-1}\right)|\nabla \bar{x}|^{n-2} \nabla \bar{x}\right)+H_{x}^{0}(y, \bar{x})=G_{x}^{0}(y, \bar{x}), \\
\left.\bar{x}(y)\right|_{\partial \Omega}=0, x \in W_{0}^{1, n}(\Omega) .
\end{gathered}
$$


The only growth type assumption that we impose on the nonlinearity $G$, is (1.4). In the following example we show the family of functions for which G1-G3, H1-H2 are satisfied.

EXAMPLE 1.1. Let $\beta=\left(\left((\operatorname{vol}(\Omega))^{1 / m} c_{S}^{n-1}\right) /\left(M_{1}^{k}\right) C\right)^{-1}$. For $k=0,1,2 \ldots$ we put

$$
G_{k}(y, x)=\beta\left(\frac{1}{2} e^{x}-\frac{1}{8} x^{4}-f_{k}(y) x^{2}\right),
$$

where $f_{k} \in L^{\infty}(\Omega)$ with essesup $f_{k}(y) \in[1,2]$ and with $\lim _{k \rightarrow \infty} f_{k}(y)=f_{0}(y)$ for almost everywhere $y \in \Omega$ and let $n=5$. Now we show the existence of the sequence $\left\{d_{k}\right\}_{k=0}^{\infty}$ of decreasing positive numbers bounded away from 0 . We need to show that (1.4) is satisfied, that is,

$$
\max _{x \in\left[-d_{k}, d_{k}\right]}\left(\frac{1}{2} e^{x}-\frac{1}{2} x^{3}-f_{k}(y) x\right) \leqslant d_{k}^{m-1} .
$$

The above inequality is satisfied for $d_{k} \leqslant 18$. The remaining assumptions obviously hold. As far as function $H$ is concerned we may take any function $H^{k}(y, x)=f_{k}(y) x^{2}+h_{k}(x)$, where $f_{k} \in L^{\infty}(\Omega)$ for $k=0,1,2 \ldots, f_{k}(y) \geqslant 0$ and $h_{k}: R \rightarrow R$ is a convex continuously differentiable function such that $h_{k}^{\prime}(x) x \geqslant 0$ for all $x \in R$.

In the paper we construct a variational method which is derived by considering the action and a dual action functionals connected with the investigated equation. The relations between both functionals are obtained with the aid of a Fenchel-Young conjugacy, see $[\mathbf{1}, \mathbf{8}]$. In order to provide an approach that will apply not only for Dirichlet problem (1.1) but also for some other type of Dirichlet problems we write a duality theory for some more general Dirichlet problem. In this paper we substantially modify the variational method form [2] and also provide the possibility of considering the differential operator varying with $k$. In order to prove existence and stability results we investigate relations between the critical values and critical points of a certain type to the primal and dual action functional, see formulas (2.4) and (2.5). The idea of stability of solutions for the Dirichlet problems which we apply originates mainly from work [9]. Although it is the approach of the author's previous work [2] that we use.

By other methods, that is, by mountain pass approach or some other differential or topological approach, the Dirichlet problems of elliptic type are considered for example in $[3,4,5,7]$. A dual variational method is applied [6] but for less general differential operator that does not depend on a numerical parameter. The existence results in the papers cited differ substantially from ours. 


\section{Duality Results and the Existence of Solutions}

We shall develop the existence results for the following family of Dirichlet Problems for $k=0,1,2, \ldots$

$$
\begin{gathered}
-\operatorname{div} F_{z}^{k}(y, \nabla x(y))+H_{x}^{k}(y, x)=G_{x}^{k}(y, x) \\
x\left(\left.y\right|_{\partial \Omega}=0, x \in W_{0}^{1, n}(\Omega)\right.
\end{gathered}
$$

where we denote $-\varphi_{k}\left(y,|\nabla x|^{n-1}\right)|\nabla x|^{n-2} \nabla x$ by $F_{z}^{k}(y, \nabla x(y))$ and by $F^{k}: \Omega \times R^{r} \rightarrow R$ we denote its potential. We first fix $k=0,1,2, \ldots$ and consider the following set

$$
\begin{aligned}
\tilde{X}_{k}=\left\{x \in W_{0}^{1, n}(\Omega), x(y) \in\left[-d_{k}, d_{k}\right] \text { on } \Omega,\|\nabla x\|_{L^{n}(\Omega)}^{n-1} \leqslant C \frac{(\operatorname{vol}(\Omega))^{1 / m}}{M_{1}^{k}} g_{k}\right. \\
\left.\quad-\operatorname{div}\left(\varphi_{k}\left(\cdot,|\nabla x(\cdot)|^{n-1}\right)|\nabla x(\cdot)|^{n-2} \nabla x(\cdot)\right)+H_{x}^{k}(\cdot, x(\cdot)) \in L^{\infty}(\Omega, R)\right\}
\end{aligned}
$$

LEMma 2.1. Assume G1, G2, H1. For any $u \in \widetilde{X}_{k}$ there exists a solution $x \in \widetilde{X}_{k}$ to Dirichlet Problem

$$
-\operatorname{div}\left(\varphi_{k}\left(y,|\nabla x|^{n-1}\right)|\nabla x|^{n-2} \nabla x\right)+H_{x}^{k}(y, x)=G_{x}^{k}(y, u), x\left(\left.y\right|_{\partial \Omega}=0 .\right.
$$

Proof: Indeed, we take any $u \in \tilde{X}_{k}$. We show the existence of solution to (2.2) by a direct variational method, [5]. Indeed the action functional

$$
J_{A}^{k}(x)=\int_{\Omega} F^{k}(y, \nabla x(y)) d y+\int_{\Omega} H^{k}(y, x(y)) d y-\int_{\Omega} G^{k}(y, u(y)) x(y) d y
$$

for which (2.2) is the Euler-Lagrange equation is by (1.2) and by (1.6) and by convexity of $H$ coercive on $W_{0}^{1, n}(\Omega)$. Being convex it is also weakly lower semicontinuous. Hence the action functional $J_{A}$ has a minimum over $W_{0}^{1, n}(\Omega)$ which satisfies its Euler-Lagrange equation, that is, (2.2). By (1.4) we obtain

$$
-\operatorname{div}\left(\varphi_{k}\left(\cdot,|\nabla x(\cdot)|^{n-1}\right)|\nabla x(\cdot)|^{n-2} \nabla x(\cdot)\right)+H_{x}^{k}(\cdot, x(\cdot)) \in L^{\infty}(\Omega, R) .
$$

Hence

$$
\begin{aligned}
\int_{\Omega}-\operatorname{div}\left(\varphi_{k}\left(y,|\nabla x(y)|^{n-1}\right)|\nabla x(y)|^{n-2} \nabla x(y)\right) x(y)+ & H_{x}^{k}(y, x(y)) x(y) d y \\
& =\int_{\Omega} G_{x}^{k}(y, u(y)) x(y) d y .
\end{aligned}
$$

Therefore using definition of $g_{k}$, see (1.9), inequality (1.3) and (1.2) we get

$$
\begin{aligned}
M_{1}^{k} \int_{\Omega}|\nabla x(y)|^{n}+H_{x}^{k}(y, x(y)) x(y) d y & \leqslant \int_{\Omega} \varphi_{k}\left(y,|\nabla x(y)|^{n-1}\right)|\nabla x(y)|^{n} \\
& +H_{x}^{k}(y, x(y)) x(y) d y \leqslant C g_{k} \sqrt[n]{\int_{\Omega}|\nabla x(y)|^{n} d y} .
\end{aligned}
$$


Since $\int_{\Omega} H_{x}^{k}(y, x(y)) x(y) d y \geqslant 0$ and by (1.4) we obtain

$$
\|\nabla x\|_{L^{n}(\Omega)}^{n-1} \leqslant C \frac{(\operatorname{vol}(\Omega))^{1 / m}}{M_{1}^{k}} g_{k}
$$

Therefore and by (1.4)

$$
\|x\|_{C(\Omega)}^{n-1} \leqslant C \frac{(\operatorname{vol}(\Omega))^{1 / m} c_{S}^{n-1}}{M_{1}^{k}} g_{k} \leqslant d_{k}^{n-1} .
$$

Thus $x(y) \in\left[-d_{k}, d_{k}\right]$ for all $y \in \Omega$.

Therefore we may for each $k=0,1,2, \ldots$ define the $X_{k} \subset W^{1, p}(\Omega)$ in the following manner: for each $x \in X_{k}$ the relation

$$
\begin{gathered}
-\operatorname{div} F_{z}^{k}(y, \nabla \widetilde{x}(y))+H_{x}^{k}(y, \widetilde{x})=G_{x}^{k}(y, x) \\
\left.\tilde{x}(y)\right|_{\partial \Omega}=0, \tilde{x} \in W_{0}^{1, n}(\Omega)
\end{gathered}
$$

implies $\widetilde{x} \in X_{k}$.

In view of Lemma 2 we may put $X_{k}=\tilde{X}_{k}$. By its definition $X_{k}$ is weakly compact in $W^{1, p}(\Omega)$.

Let $W=\left\{p \in L^{q}(\Omega): \operatorname{div} p \in L^{q}(\Omega)\right\}$. The functional $J_{k}: W^{1, p}(\Omega) \rightarrow R$ for which (2.1) for $k=0,1,2 \ldots$ is the Euler-Lagrange equation reads

$$
J_{k}(x)=\int_{\Omega} F^{k}(y, \nabla x(y)) d y+\int_{\Omega} H^{k}(y, x(y)) d y-\int_{\Omega} G^{k}(y, x(y)) d y
$$

and the dual functional $J_{D_{k}}: W \times L^{q}(\Omega) \rightarrow R$ is given by

$$
\begin{aligned}
J_{D_{k}}(p, q)=\int_{\Omega}\left(G^{k}\right)^{*}(y,-\operatorname{div} p(y) & +q(y)) d y \\
& -\int_{\Omega}\left(H^{k}\right)^{*}(y, q(y)) d y-\int_{\Omega}\left(F^{k}\right)^{*}(y, p(y)) d y .
\end{aligned}
$$

For each $k=0,1,2, \ldots$ we now define a set $X_{k}^{d}$ which comprises of these $(p, q) \in W$ $\times L^{q}(\Omega)$ for which there exists an $x \in X_{k}$ such that

$$
p(y)=F_{z}^{k}(y, \widetilde{x}(y)), q(y)=H_{x}^{k}(y, \widetilde{x}(y))
$$

almost everywhere and where $\tilde{x} \in X$ is related to $x$ by definition of $X_{k}$, see relation (2). By its definition it follows that $X_{k}^{d}$ is nonempty and relatively weakly compact in $W \times L^{q}(\Omega)$. 
Theorem 2.2. Assume G1, G2, H1. For any $k=0,1,2, \ldots$ there exists a triple $\left(\bar{x}_{k}, \bar{p}_{k}, \bar{q}_{k}\right) \in W^{1, p}(\Omega) W \times L^{q}(\Omega)$ satisfying the system

$$
\begin{aligned}
\inf _{(p, q) \in X_{k}^{d}} J_{D_{k}}(p, q) & =J_{D_{k}}\left(\bar{p}_{k}, \bar{q}_{k}\right)=J_{k}\left(\bar{x}_{k}\right)=\inf _{x \in X_{k}} J_{k}(x), \\
\bar{p}_{k}(y) & =F_{z}^{k}\left(y, \bar{x}_{k}(y)\right), \\
\bar{q}_{k}(y) & =H_{x}^{k}\left(y, \bar{x}_{k}(y)\right), \\
-\operatorname{div} \bar{p}_{k}(y)+\bar{q}_{k}(y) & =G_{x}^{k}\left(y, \bar{x}_{k}(y)\right) .
\end{aligned}
$$

Proof: Let us fix $k=0,1,2, \ldots$. We observe that $J_{k}$ is bounded from below on $X_{k}$. Thus and by the properties of $X_{k}$ there exists a minimising sequence $\left\{x_{k}^{j}\right\}_{j=1}^{\infty}$ for $J_{k}$ which may be assumed to be weakly convergent in $W^{1, p}(\Omega)$ and thus strongly in $L^{p}(\Omega)$. Since $X_{k}$ is weakly compact, the limit $\bar{x}_{k}$ of the sequence $\left\{x_{k}^{j}\right\}_{j=1}^{\infty}$ belongs to $X_{k}$. Since $J_{k}$ is weakly lower semicontinuous $X_{k}$ we obtain $J_{k}\left(\bar{x}_{k}\right)=\inf _{x \in X_{k}} J_{k}(x)$. In order to demonstrate that system (2.6)-(2.9) is satisfied, in other words to show that equation (2.1) has a solution, we shall have to construct a duality theory relating both action functionals. First we relate the critical values to both functionals on sets $X_{k}, X_{k}^{d}$ and later by showing that there exists $\left(\bar{p}_{k}, \bar{q}_{k}\right) \in X_{k}^{d}$ corresponding to $\bar{x}_{k}$ we have system (2.6)-(2.9) satisfied.

We begin with demonstrating

$$
\inf _{(p, q) \in X_{k}^{d}} J_{D_{k}}(p, q)=\inf _{x \in X_{k}} J_{k}(x) .
$$

We consider the following auxiliary functional $J_{D_{k}, \text { pert }}^{\#}: X_{k}^{d} \times X_{k} \rightarrow R$ defined by

$$
\begin{aligned}
J_{D_{k}, p e r t}^{\#}(p, q, x)= & \int_{\Omega} F^{k}(y, \nabla x(y)) d y+\int_{\Omega} H^{k}(y, x(y)) d y \\
& +\int_{\Omega}\left(G^{k}\right)^{*}(y,-\operatorname{div} p(y)+q(y)) d y-\int_{\Omega}(-\operatorname{div} p(y)+q(y)) x(y) d y
\end{aligned}
$$

First we prove that for any $(p, q) \in X_{k}^{d}$

$$
\inf _{x \in X_{k}} J_{D_{k}, p e r t}^{\#}(p, q, x)=J_{D_{k}}(p, q)
$$

Fix $(p, q) \in X_{k}^{d}$ and observe that by the Fenchel-Young inequality and the definition of the Fenchel-Young transform for convex integral functionals, see [8], we obtain

$$
\int_{\Omega}(-\operatorname{div} p(y)) x(y) d y-\int_{\Omega} F^{k}(y, \nabla x(y)) d y \leqslant \int_{\Omega}\left(F^{k}\right)^{*}(y, p(y)) d y
$$

and

$$
\int_{\Omega} q(y) x(y) d y-\int_{\Omega} H^{k}(y, x(y)) d y \leqslant \int_{\Omega}\left(H^{k}\right)^{*}(y, q(y)) d y
$$


for all $x \in X_{k}$. Moreover by the definition of $X_{k}^{d}$,

$$
p(y)=F_{z}^{k}\left(y, \tilde{x}_{p}(y)\right), q(y)=H_{x}^{k}\left(y, \tilde{x}_{p}(y)\right)
$$

for certain $x_{p}, \widetilde{x}_{p} \in X_{k}$ related by (2). Therefore we get

$$
\int_{\Omega}(-\operatorname{div} p(y)) \tilde{x}_{p}(y) d y-\int_{\Omega} F^{k}\left(y, \nabla \tilde{x}_{p}(y)\right) d y=\int_{\Omega}\left(F^{k}\right)^{*}(y, p(y)) d y
$$

and

Hence

$$
\int_{\Omega} q(y) \widetilde{x}_{p}(y) d y-\int_{\Omega} H^{k}\left(y, \widetilde{x}_{p}(y)\right) d y=\int_{\Omega}\left(H^{k}\right)^{*}(y, q(y)) d y .
$$

$$
\begin{aligned}
\int_{\Omega}\left(F^{k}\right)^{*}(y, p(y)) d y+\int_{\Omega}\left(H^{k}\right)^{*}(y, q(y)) d y & \\
=\sup _{x \in X_{k}}\left\{\int_{\Omega}(-\operatorname{div} p(y)+q(y)) x(y) d y\right. & \left.-\int_{\Omega} F^{k}(y, \nabla x(y)) d y-\int_{\Omega} H^{k}(y, x(y)) d y\right\} \\
& \leqslant \int_{\Omega}\left(F^{k}\right)^{*}(y, p(y)) d y+\int_{\Omega}\left(H^{k}\right)^{*}(y, q(y)) d y .
\end{aligned}
$$

and thus (2.11) follow.

Next we prove that for any $x \in X_{k}$

$$
\inf _{(p, q) \in X_{k}^{d}} J_{D_{k}, \text { pert }}^{\#}(p, q, x)=J_{k}(x) .
$$

Fix $x \in X_{k}$. We obtain

$$
\int_{\Omega}(-\operatorname{div} p(y)+q(y)) x(y) d y-\int_{\Omega}\left(G^{k}\right)^{*}(y,-\operatorname{div} p(y)+q(y)) d y \leqslant \int_{\Omega} G^{k}(y, x(y)) d y
$$

and by definitions of $X_{k}$ and $X_{k}^{d}$ it follows that there exists $\left(p_{x}, q_{x}\right) \in X_{k}^{d}$ such that

$$
-\operatorname{div} p_{x}(y)+q_{x}(y)=G_{x}^{k}(y, x(y))
$$

This shows (2.13) by the same argument as applied above.

Now by (2.11) and (2.13) we have (2.10) since

$$
\inf _{x \in X_{k}} \inf _{(p, q) \in X_{k}^{d}} J_{D_{k}, p e r t}^{\#}(p, q, x)=\inf _{(p, q) \in X_{k}^{d}} \inf _{x \in X_{k}} J_{D_{k}, p e r t}^{\#}(p, q, x) .
$$

Since $\bar{x}_{k} \in X_{k}$, there exists $\left(\bar{p}_{k}, \bar{q}_{k}\right) \in X_{k}^{d}$ such that relation (2.9) holds. Hence and by the Fenchel-Young inequality we get $J_{k}\left(\bar{x}_{k}\right) \geqslant J_{D_{k}}\left(\bar{p}_{k}, \bar{q}_{k}\right)$. By $(2.10)$ and by $J_{k}\left(\bar{x}_{k}\right)$ $=\inf _{x \in X_{k}} J_{k}(x)$ we obtain $J_{D_{k}}\left(\bar{p}_{k}, \bar{q}_{k}\right)=J_{k}\left(\bar{x}_{k}\right)$ and (2.6) follows. Thus we have

$$
\begin{aligned}
& \int_{\Omega}\left(F^{k}\right)^{*}\left(y, \bar{p}_{k}(y)\right) d y+\int_{\Omega} F^{k}\left(y, \nabla \bar{x}_{k}(y)\right) d y-\int_{\Omega} \bar{p}_{k}(y) \nabla \bar{x}_{k}(y) d y \\
& \int_{\Omega} H^{k}\left(y, \bar{x}_{k}(y)\right) d y+\int_{\Omega}\left(H^{k}\right)^{*}\left(y, \bar{q}_{k}(y)\right) d y-\int_{\Omega} \bar{q}_{k}(y) \bar{x}_{k}(y) d y=0 .
\end{aligned}
$$

Since by the Fenchel-Young inequalities both terms are nonnegative we get (2.8) and (2.7) satisfied. 


\section{The Stability of Solutions}

THEOREM 3.1. We assume G1, G2, G3, H1, H2. Let $G_{x}^{k}(y, x) \underset{k \rightarrow \infty}{\rightarrow} G_{x}^{0}(y, x)$, $H_{x}^{k}(y, x) \underset{k \rightarrow \infty}{\rightarrow} H_{x}^{0}(y, x), \varphi_{k}(y, x) \underset{k \rightarrow \infty}{\rightarrow} \varphi_{0}(y, x)$ almost everywhere on $\Omega$ for any $x \in\left[-d_{0}, d_{0}\right]$. For each $k \in N$ there exists a solution $x_{k}$ to the problem (1.1) and there exists a subsequence $\left\{x_{k_{j}}\right\}_{j=1}^{\infty}$ and $\bar{x} \in X_{0}$ such that $\lim _{j \rightarrow \infty} x_{k_{j}}=\bar{x}$ weakly in $W_{0}^{1, n}(\Omega)$ and

$$
\begin{gathered}
-\operatorname{div}\left(\varphi_{0}\left(y,|\nabla \bar{x}|^{n-1}\right)|\nabla \bar{x}|^{n-2} \nabla \bar{x}\right)+H_{x}^{0}(y, \bar{x})=G_{x}^{0}(y, \bar{x}), \\
\left.\bar{x}(y)\right|_{\partial \Omega}=0 .
\end{gathered}
$$

Proof: By Theorem 2 for each $k=1,2, \ldots$ there exists a solution to (1.1). Since $d_{k} \leqslant d_{0}$ for $k=1,2, \ldots$ it follows that $X_{k} \subset X_{0}$ for all $k=1,2, \ldots$. There exists a subsequence $\left\{x_{k_{j}}\right\}_{j=1}^{\infty}$ of $\left\{x_{k}\right\}_{k=1}^{\infty}$ weakly convergent in $W_{0}^{1, n}(\Omega)$, which up to subsequence may be assumed to be strongly convergent $\bar{x} \in X_{0}$ in $L^{n}(\Omega)$.

We now prove that

$$
\lim _{n \rightarrow \infty} G_{x}^{k_{j}}\left(y, x_{k_{j}}(y)\right)=G_{x}^{0}(y, \bar{x}(y)) \text { almost everywhere, }
$$

possibly up to a subsequence. Indeed, we get

$$
\begin{aligned}
G_{x}^{k_{j}}\left(y, x_{k_{j}}(y)\right)- & G_{x}^{0}(y, \bar{x}(y)) \\
& =G_{x}^{k_{j}}\left(y, x_{k_{j}}(y)\right)-G_{x}^{k_{j}}(y, \bar{x}(y))+G_{x}^{k_{j}}(y, y, \bar{x}(y))-G_{x}^{0}(y, \bar{x}(y)) .
\end{aligned}
$$

By (1.5) and by the mean value theorem we observe that

$$
\left|G_{x}^{k_{j}}\left(y, x_{k_{j}}(y)\right)-G_{x}^{k_{j}}(y, \bar{x}(y))\right| \leqslant a\left|x_{k_{j}}(y)-\bar{x}(y)\right| .
$$

Since $\left\{x_{k_{j}}\right\}_{n=1}^{\infty}$ is convergent almost everywhere it follows that

$$
\lim _{n \rightarrow \infty}\left(G_{x}^{k_{j}}\left(y, x_{k_{j}}(y)\right)-G_{x}^{k_{j}}(y, \bar{x}(y))\right)=0 .
$$

Thus from (3.3) using the above and the assumptions we obtain (3.2). By the same arguments we demonstrate that

$$
\lim _{n \rightarrow \infty} H_{x}^{k_{j}}\left(y, x_{k_{j}}(y)\right)=H_{x}^{0}(y, \bar{x}(y)) \text { almost everywhere. }
$$

By (1.4), (1.7) and by $O 1$ we infer that $-\operatorname{div}\left(\varphi_{k}\left(\cdot,|\nabla x(\cdot)|^{n-1}\right)|\nabla x(\cdot)|^{n-2} \nabla x(\cdot)\right)$ is bounded in $L^{\infty}(\Omega)$. Hence it is weakly convergent in $L^{m}(\Omega)$, up to a subsequence, to a certain $d \in L^{m}(\Omega)$. We obtain by monotonicity and proceeding considerations for any 


$$
\begin{aligned}
& x \in W_{0}^{1, n}(\Omega) \\
& 0 \leqslant \int_{\Omega}\left\langle-\operatorname{div}\left(\varphi_{k}\left(y,\left|\nabla x_{k_{j}}\right|^{n-1}\right)\left|\nabla x_{k_{j}}\right|^{n-2} \nabla x_{k_{j}}\right)\right. \\
& \left.+\operatorname{div}\left(\varphi_{k}\left(y,|\nabla x|^{n-1}\right)|\nabla x|^{n-2} \nabla x\right), x_{k_{j}}-x\right\rangle \\
& \rightarrow \int_{\Omega}\left\langle d(y)+\operatorname{div}\left(\varphi_{k}\left(y,|\nabla x|^{n-1}\right)|\nabla x|^{n-2} \nabla x\right), \bar{x}-x\right\rangle .
\end{aligned}
$$

Hence $d(y)=-\operatorname{div}\left(\varphi_{k}\left(y,|\nabla \bar{x}(y)|^{n-1}\right)|\nabla \bar{x}(y)|^{n-2} \nabla \bar{x}(y)\right)$.

Since a weak limit is equal to an almost everywhere limit we finally get (3).

\section{REFERENCES}

[1] I. Ekeland and R. Temam, Convex analysis and variational problems (North-Holland, Amsterdam, 1976).

[2] M. Galewski, 'Existence, stability and approximation of solutions for a certain class of Nonlinear BVP's', Nonlinear Anal. 65 (2006), 159-174.

[3] D. Gilbarg and N.S. Trudinger, Elliptic partial differential equations of second order, Classics in Mathematics, (Reprint of the 1998 edition) (Springer-Verlag, Berlin, 2001).

[4] P. Magrone, 'On a class of semilinear elliptic equations with potential changing sign', Dynam. Systems Appl. 9 (2000), 459-467.

[5] J. Mawhin, Problems de Dirichlet variationnels non lineaires (Les Presses de l'Universite de Montreal, Canada, 1987).

[6] A. Orpel, 'On the existence of positive solutions and their continuous dependence on functional parameters for some class of elliptic problems', J. Differential Equations 204 (2004), 247-264.

[7] P.H. Rabinowitz, Minimax methods in critical point theory with applications to differential equations, CBMS, Regional Conference Series in Mathematics 65 (American Mathematical Society, Providence, RI, 1986).

[8] R.T. Rockafellar, Contributions to Nonlinear Functional Analysis, in, (E. Zarantonello, Editor) (Academic Press, New York, 1971), pp. 215-236.

[9] S. Walczak, 'On the continuous dependance on parameters of solutions of the Dirichlet problem. Part I Coercive Case, Part II. The Case of Saddle Points', Bulletin de la Case des Sciences de l'Academie Royale de Beligique 6 (1995), 247-273.

Faculty of Mathematics,

University of Lodz,

Banacha 22

90-238 Lodz

Poland,

e-mail: galewski@math.uni.lodz.pl 